\title{
Poor glycemic control enhances the disease activity in the RA patients with undiagnosed diabetes - a cross-sectional clinical study
}

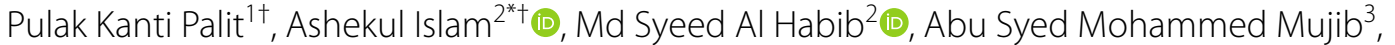 \\ Joyonti Datta', Bahni Chakraborty', Suman Dhar', Dhiman Chowdhury' , Md Faisal Fahad Chowdhury', \\ Mohammad Shawkat Ali', Arfana Yesmin', Mohammod Johirul Islam², Nishat Akther ${ }^{2}$, Abdullah Al Mamun ${ }^{2}$, \\ Khairul Islam² and Pradip Kumar Nath ${ }^{1}$
}

\begin{abstract}
Background: Rheumatoid arthritis (RA), an autoimmune disorder, characterized by systemic inflammation and swollen joints, establishes itself as a critical threat. A pro-inflammatory cytokine TNF-a is a well-known driver of RA pathogenesis and at the same time predisposes to insulin resistance through signal impediment which ultimately paves the way for type 2 diabetes (T2DM). However, in patients with RA, T2DM remains significantly undiagnosed or undertreated, apparently which increases the risk of developing cardio-metabolic comorbidities. This study aimed to evaluate the glycemic status among RA patients and its association with disease activity.

Result: One hundred fifty inpatients RA cases according to ACR/EULAR standards were included in the cross-sectional study who have an average age of $45.4 \pm 12.15$ years and a median and interquartile period of RA of 2.25 years and $0.48-6$ years, respectively. We discovered that $36 \%$ of people had T2DM, 26\% were prediabetic, and 38\% were non-diabetic. Age was shown to be significantly correlated with DM frequency in RA patients $(p=0.007)$. There were 28 patients with elevated disease activity (19\%) and 60 patients with low disease activity (40\%) in this study. No substantial associations were found in the presence of DM with gender, anti-CCP, RF, disease duration, or DAS28.

Conclusion: RA patients are more likely to experience diabetes, and resultantly a high index of notion must be kept. Clinician should be aware about the affliction of undiagnosed diabetes and prediabetes in RA patients. Furthermore, keeping an eye on glycemic control in RA patients could prevent metabolic and cardiovascular comorbidities in those susceptible patients.
\end{abstract}

Keywords: Diabetes mellitus, Rheumatoid arthritis, Glycemic control, DAS-28, RF, Anti-CCP, Biochemical parameter

\section{Background}

With a prevalence of $0.5-1 \%$ worldwide, rheumatoid arthritis (RA) is a progressive inflammatory autoimmune condition that predominantly affects the lining of synovial joints and is marked by articular and sometimes extra-articular

\footnotetext{
*Correspondence: ashekbmb@mbstu.ac.bd

${ }^{\dagger}$ Pulak Kanti Palit and Ashekul Islam contributed equally to this work.

${ }^{2}$ Mawlana Bhashani Science and Technology University, Santosh,

Tangail 1902, Bangladesh

Full list of author information is available at the end of the article
}

manifestations [1]. It is associated with suffering, gradual disability, which leads to reduced quality of life or longevity [2]. The overall standardized mortality ratio (SMR) has been estimated at 2.26 and increased with time and hence, as contrasted with the typical individual in the country, a person with RA would be twice as likely to die at the same age. Mortality of the RA-patient is strikingly increased for specific causes including infection, lymphoproliferative malignancy, or gastroenterological problems. Furthermore, as a result of SMR 2.26, the number of predicted deaths rises 
in all causes (except cancer) without any particular reference to the extent of deaths due to cardiovascular and cerebrovascular diseases. Age, education level, male sex, rheumatoid factor, nodules, erythrocyte sedimentation rate, joint count, and prednisone uses are predictors which improve death. Moreover, management of patients with RA is costly; Maetzel and colleagues estimated that the annual expense of RA therapy is about double that of osteoarthritis (OA) [3].

RA should be diagnosed and managed promptly to prevent the sharp progression and possible treatment. The ultimate objective of therapy is the prevention or management of joint destruction and consequent functional loss [4]. The long-term RA prognosis has greatly increased after the advent of extremely efficient disease-modifying anti-rheumatic drugs (DMARD) and improved management practices, such as tightened regulation and treatto-target $[5,6]$. However, various co-morbidities may influence the long-term prognosis, which results in a rise in mortality rate in contrast to the overall population. The leading factors of excess mortality in RA patients include cardiovascular disease, infections, and certain kinds of malignancies [7-9]. Notably, comorbidities may be attributed to the disease behavior itself or arise as a consequence of treatment.

Due to insulin secretion, defects in action, or to both causing changes in the absorption of carbohydrates, fat and protein bring about a metabolic syndrome which is known as Diabetes mellitus (DM). The overwhelming majority of the cases of DM consist of type I and type II, distinguished by total insulin insufficiency and insulin insensitivity with partial deficiency [10]. The incidence of DM is unclear in RA but most prior data suggest that DM or insulin resistance in RA has risen because of activation of the immune system and/or RA therapy $[11,12]$. Several epidemiological studies showed that rheumatoid arthritis (RA) is linked with the premature development of cardiovascular disease [13-16]. This is related, at least in part, to the systemic inflammatory load in RA which has been shown to predispose people with this disorder to the development of premature atherosclerosis. Increased amounts of systemic inflammation were also shown to be predisposed to both insulin resistance [1618] and type 2 diabetes mellitus (T2DM) [19-21].

Pradhan and colleagues reported that the development of T2DM in women was predicted by elevated levels of C-reactive protein (CRP) and interleukin [16], which are markers of systemic inflammation [19]. In the cohort studies, the authors found that inflammation markers such as CRP, raised white cell count, and low serum albumin was associated with the development of diabetes over prolonged periods [20,21]. Svenson et al. reported that the possible effect of insulin resistance in RA patients is a cardiovascular risk factor, and controls have been compared with reduced glucose metabolism in patients with inflammatory arthritis [22]. An inverse association between insulin sensitivity and acute phase markers in RA has also been demonstrated by the same group. Previously, when patients with inflammatory arthritis compared with healthy control, Dessein, et al. evinced significantly higher levels of insulin resistance are correlated with elevated CRP levels [23, 24]. Moreover, tumor necrosis factor-alpha (TNF- $\alpha$ ), a notable pro-inflammatory mediator in RA, promoting beta-cell malfunction followed by insulin resistance eventually causes bone damage $[25,26]$. However, TNF inhibitors minimize the likelihood that DM can evolve in RA patients, indicating that TNF plays an important role in RA and DM pathogenesis [27]. Besides, RA causes joints to become stiff, swollen, and agonizing. As a result, the patient may not want to exercise or move. One study found that $42 \%$ of people with RA are not physically active. Not staying active raises the risk of developing T2DM [28]. Moreover, T2DM co-occurrence is correlated with elevated incidence of cardiovascular disease in patients with RA [16].

To our knowledge, there is no such study that has been performed in our country. Taking the potential link between DM and RA into consideration, prior detection of DM will significantly increase prolonged prognosis and therefore decrease the economic burden of RA patients and death rate. The aim of this research was to examine non-diabetic RA's glycemic status and to assess the relationship between RA and DM's clinical characteristics as well as the therapy response in RA patients with diagnosed DM.

\section{Methods}

A hospital-based descriptive cross-sectional study was conducted among the patients diagnosed with RA admitted at the Inpatient Department and visited OPD in Medicine Department of Chittagong Medical College Hospital (CMCH), Chittagong-4203, Bangladesh, from 1st January 2020 to 30th June 2020 attending outpatient or inpatient care.

\section{Inclusion criteria}

ACR/EULAR standards were utilized upon patients to diagnose with RA. Detailed history was recorded from all patients with special reference to the duration of pain or inflammation. Both sexes and age above 16 years were included throughout the study.

\section{Exclusion criteria}

Other connective tissue rheumatic diseases, known diabetic or prediabetic, diabetic cheiroarthropathy, heart failure or stroke, acute coronary syndromes, CVD, pregnancy, encephalopathy, CKD, bleeding disorder, hypothyroidism, 
hyperthyroidism, and subjects were excluded from this study who did not provide written consent to participate.

\section{Procedure of the work}

As RA patients were included, an approval from the Bangladesh College of Physicians and Surgeons (BCPS) was taken prior to the start of this study. Informed written consents were taken after explaining the purpose and procedure of the study from the patient or guardian of the patients. They were assured that unwillingness to participate in the study would not hamper the ongoing treatment. After getting consent, clinical history and physical examination were done. Before the examination, the subject or the attendant of the subject was fully briefed regarding the goals, objectives, and detailed protocol of the study. She/he was motivated to volunteer and given freedom to withdraw anytime she/he wished, even after participation. Blood sample was collected and sent for biochemical analysis.

\section{Data collection and analysis}

A pre-designed semi-structured case record form was used to collect information. After collection data were compiled in a Microsoft Office Excel Worksheet. Then that were fed into either SPSS (Statistical Package for Social Science) for Windows version 23 software or Graph Pad Prism (Version 7.01, USA) to analyze the data. Continuous variables were reported as the means \pm SD or median (interquartile range) depending upon their distributions and categorical variables were reported as frequency (percentages). Student's $t$ test or Mann-Whitney $U$ test was used to compare continuous data. Chi-square or Fisher's exact test was used to compare proportions. Correlation coefficient was measured to evaluate the correlation between variables. Receiver operating characteristic (ROC) curves were built to evaluate prophesy of selected variables on the likelihood of being classified as diabetes. Statistical significance was defined as $P \leq 0.05$ and confidence interval (CI) set at $95 \%$ level. Study flow chart

\section{RA patients attending in medicine outdoor of $\mathrm{CMCH}$ were assessed for eligibility}
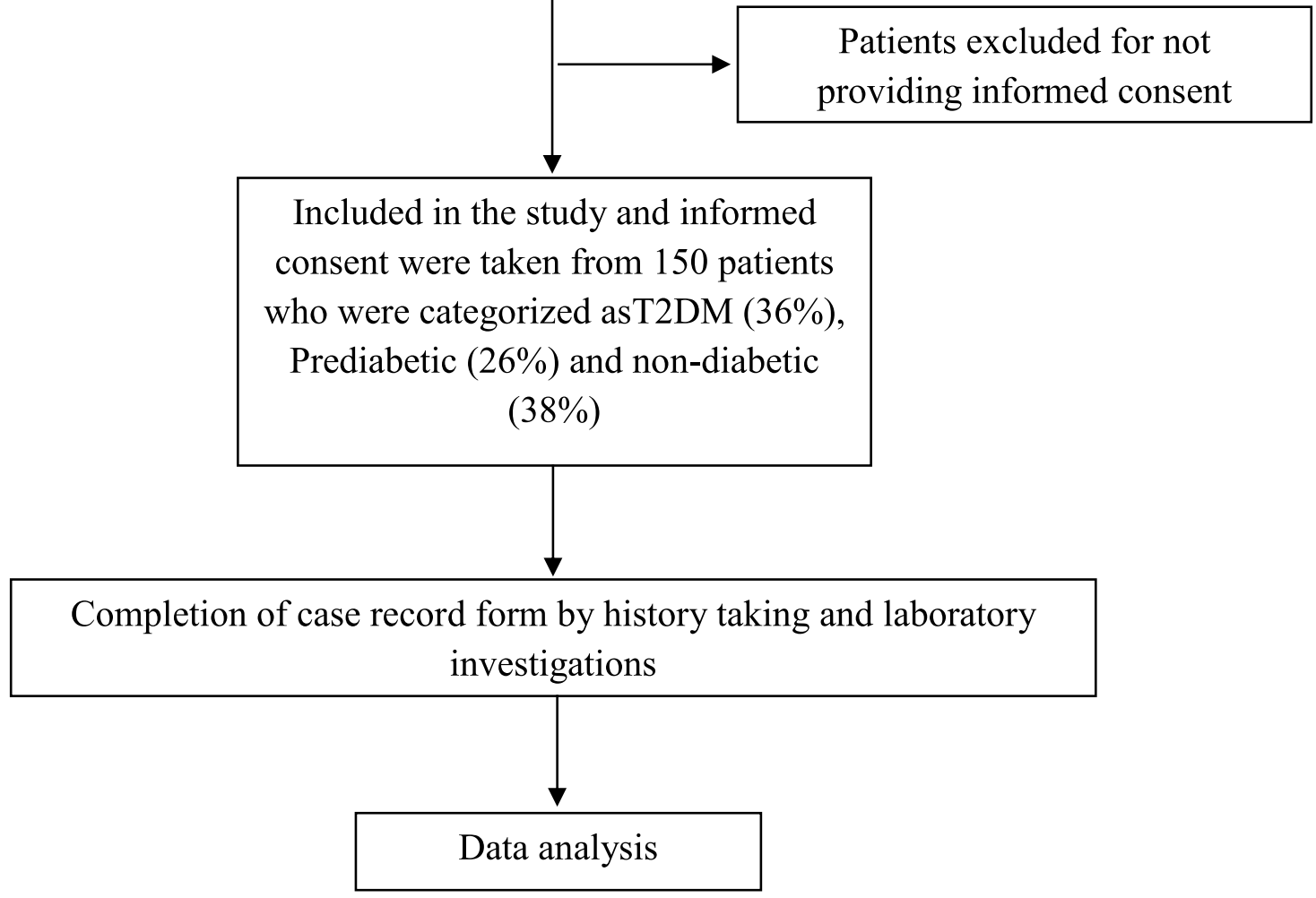


\section{Results}

The study was conducted intensively in the Medicine Department of $\mathrm{CMCH}$, among the patients with rheumatoid arthritis (RA). To identify the glycemic status fasting blood sugar (FBS), blood sugar, postprandial glucose (PPG) test, and hemoglobin A1c test (HbA1C) were done and disease severity was measured by the DAS- 28 scale. The findings of the study are summarized in the following tables and graphs.

Table 1 shows the socio-demographic distribution of the patients. There is female predominance, and most of them had educational qualification below or up to SSC.

The study showed most of the patients were RF and anticyclic citrullinated peptide antibodies (ACPA) positive. All of them were taking MTX and $48 \%$ were on steroids during data collection. The median disease duration was 2.25 years (IQR: $0.48-6.0$ years) (Table 2).

To assess the disease activity, the Disease Activity Score Including 28 Joints and erythrocyte sedimentation rate (DAS-28-ESR) scale was used. The results are summarized in Table 3. Most of the patients (56\%) have high disease activity (score > 5.1).

Out of 150 RA patients, 54 (36\%) were found to be diabetic either by blood sugar level or HbA1C. Summarized measures of the parameters of glycemic status are described in Table 4.

Glycemic status of the patients based on oral glucose tolerance test (OGTT) and HbA1C is presented in Fig. 1. It shows that out of 150 RA patients, 57 (38\%) participants have normal glycemic status, 39 (26\%) are prediabetic, and $54(36 \%)$ are diabetic. Although different parameters of glycemic status showed a positive correlation with disease activity (DAS-28), only the relation between FBS and DAS-28 was statistically significant (Table 5 and Fig. 2)

Table 1 Socio-demographic characteristics of the patients $(n=150)$

\begin{tabular}{lll}
\hline Variables & & \\
\hline Age (years) & Mean \pm SD & $45.4 \pm 12.15$ \\
Sex & Range & $20-72$ \\
& Male & $18(12 \%)$ \\
& Female & $132(88 \%)$ \\
Education & Male:Female & $1: 7.33$ \\
& Illiterate & $39(26 \%)$ \\
& Primary & $42(28 \%)$ \\
Monthly family income & SSC & $36(24 \%)$ \\
(BDT) & MSC and above & $33(22 \%)$ \\
& Interquartile range & 18,000 \\
\hline
\end{tabular}

Data are expressed as frequency and percentage if not otherwise mentioned $B D T$ Bangladeshi taka, $S D$ standard deviation
Table 2 Disease characteristics of the RA patients $(n=150)$

\begin{tabular}{lll}
\hline Variables & & \\
\hline Disease duration (years) & Median (IQR) & $2.25(0.48-6.0)$ \\
Rheumatoid factor & Positive & $132(88 \%)$ \\
& Negative & $18(12 \%)$ \\
ACPA & Positive & $99(66 \%)$ \\
& Negative & $51(34 \%)$ \\
Current medication & Methotrexate & $150(100 \%)$ \\
& Hydroxychloroquine & $18(12 \%)$ \\
& Steroid & $72(48 \%)$ \\
& Sulphasalazine & $27(18 \%)$ \\
Dose of steroid (mg/day) & Median (IQR) & $5(5-6.88)$ \\
Duration of current steroid & Median (IQR) & $30(28.5-42)$ \\
(intake/days) & &
\end{tabular}

Data are expressed as frequency and percentage if not otherwise mentioned

IQR interquartile range, ACPA anticyclic citrullinated peptide antibodies

We performed a ROC curve in order to investigate the prediction of the mean value of DAS-28 on the likelihood of being diagnosed with T2DM (Fig. 3). The area under the ROC curve was 0.654 (95\% CI 0.494-0.815, $P \leq 0.069)$ for the mean value of DAS-28. The analysis of the ROC curve showed that the best cut-off for mean DAS-28 was 4.78 and provided a sensitivity of $73.78 \%$ and a specificity of $46.48 \%$. Univariate analysis was used to evaluate differences between diabetic and nondiabetic groups of RA patients (Table 6). The groups differed significantly for age $(P \leq 0.007)$. On the other hand, though disease duration, TJC, SJC, and DAS-28 are higher in the diabetic group than the non-diabetic group; the differences were not statistically significant.

A proportion of T2DM and GIDM among 54 RA patients having DM is presented in Fig. 4. Our study shows that out of 54 DM patients, a total of $30(55.6 \%)$ and 24 (44.4\%) have GIDM T2DM, respectively.

Table 3 Disease activity of the study patients $(n=150)$

\begin{tabular}{lll}
\hline Variables & & \\
\hline ESR (mm in 1st hour) & Median (IQR) & $34(25-75)$ \\
C-reactive protein (CRP) & Median (IQR) & $20(6.63-48)$ \\
DAS-28-ESR & Mean ( \pm SD) & $5.55( \pm 1.39)$ \\
& Range & $3-8.18$ \\
Disease activity (category) & Remission & $0(0 \%)$ \\
& Low & $2(4 \%)$ \\
& Moderate & $20(40 \%)$ \\
& High & $28(56 \%)$ \\
\hline
\end{tabular}

Data are expressed as frequency and percentage if not otherwise mentioned IQR interquartile range, SD standard deviation, DAS-28-ESR Disease Activity Score Including 28 Joints and F 
Table 4 Blood sugar level and $\mathrm{HbA1C}$ measurements of the study patients $(n=150)$

\begin{tabular}{lll}
\hline Variables & & \\
\hline FBS (mmol/L) & Median (IQR) & $5.92(4.67-8.37)$ \\
$\mathbf{2}$ HA glucose load (mmol/L) & Median (IQR) & $7.9(6.4-12.9)$ \\
HbA1C (\%) & Median (IQR) & $5.9(5.4-6.8)$ \\
OGTT (category) & NGT & $57(38 \%)$ \\
& IFG & $15(10 \%)$ \\
& IGT & $9(6 \%)$ \\
& Both IFG and IGT & $15(10 \%)$ \\
& T2DM & $54(36 \%)$ \\
\hline
\end{tabular}

Data are expressed as frequency and percentage if not otherwise mentioned IQR interquartile range, IFG impaired fasting glucose, IGT impaired glucose tolerance, NGT normal glucose tolerance, T2DM type 2 diabetes mellitus, HbA1C hemoglobin A1c test, OGTT oral glucose tolerance test

\section{Discussions}

This hospital-based descriptive cross-sectional study was conducted to identify the prevalence of undiagnosed DM among the RA patients admitted or attending in the indoor and outdoor Medicine Department of Chittagong Medical College Hospital, Chittagong-4203, Bangladesh. In total, 150 RA patients were assessed by fasting blood sugar, blood sugar $2 \mathrm{~h}$ after glucose intake, and HbA1C. Surprisingly, our study found about 36\% of DM frequency of the total study population which is much higher than that of the study results of Emamifar and colleagues [29] who had noticed an overall prevalence of DM in their study cohort was $13 \%$. This contrasting result might be due to the differences in selection criteria or less study population. Their further study mentioned that they include only the newly diagnosed cases and are conducted in a high-resource setting. However, in previous research, there were also controversies about the

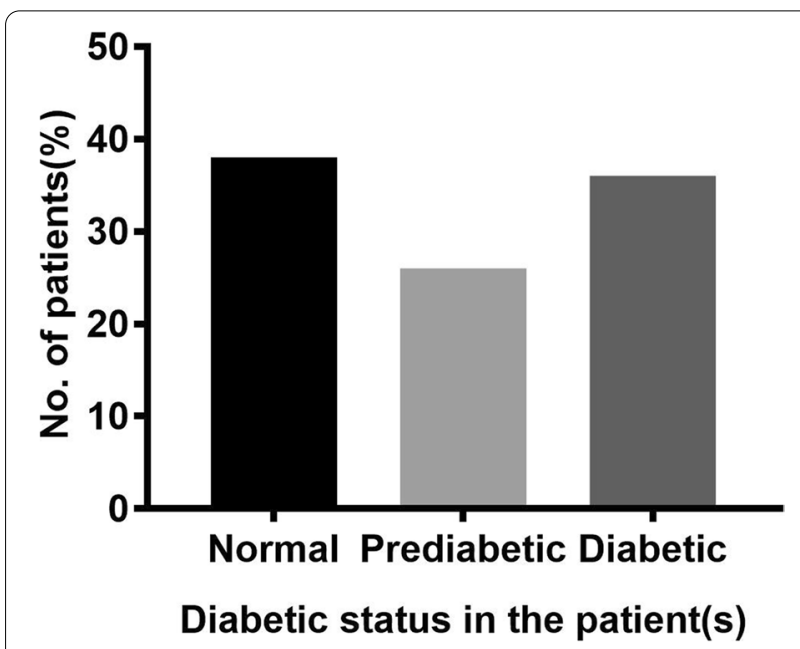

Fig. 1 Glycemic status of the patients with RA $(n=150)$
Table 5 Correlation between disease activity and different glycemic parameters among 150 RA patients

\begin{tabular}{lll}
\hline Variables & $\begin{array}{l}\text { Correlation } \\
\text { coefficient }(\boldsymbol{r})\end{array}$ & $\boldsymbol{P}$ value \\
\hline Fasting blood glucose & 0.283 & $0.046^{*}$ \\
Blood glucose 2HA post load & 0.239 & 0.094 \\
HbA1C & 0.231 & 0.106 \\
\hline
\end{tabular}

*Statistically significant

presence of DM in RA patients. Twenty-eight thousand two hundred eight RA patients of a cross-sectional analysis when compared with controls by Han $\mathrm{C}$ et al. have shown a substantially greater prevalence of DM in RA patients for their age, sex, and geographical distribution [12]. The escalated incidence of type 1 and type 2 diabetes mellitus in RA patients has been revealed by metaanalysis, involving 11 case controls and 8 cohort studies. The combined risk assessment of case-control and cohort studies showed a statistically important high incidence of DM among RA individuals [11]. Conversely, Solomon et al. failed to prove any substantial difference among female nurses having RA and who have not, regarding the prevalence of DM from a prospective longitudinal cohort analysis [30]. These conflicts may be because of the composition of the sample populations, primarily of ethnicity or other disease-related causes, e.g., disease duration.

Our current research indicates a substantial connection between age and the occurrence of DM in RA patients, which suggests that the incidence of DM rises with age. Various research, including the World Health Organization (WHO), found almost one (1) among ten (10) elderly people in Bangladesh has diabetes, which seems to be a significant public health issue, and that older people are both strongly and positively linked to prediabetes and diabetes [31]. The prevalence of prediabetes and diabetes in those between 60 and 69 years was 1.64-fold compared to younger people between the ages of 35 and 39 years; in those between the ages of 70 and older, the risk was 1.81 times higher than in the younger age category. A meta-analysis study performed by Sohail Akhter et. al. showed a relatively high prevalence of pre-diabetes and diabetes in Bangladesh [32]. Though with respect to the association between age and undiagnosed DM was in agreement with the study result of Emamifar and colleagues [29], the average age of the patients $(64.6 \pm 15.0$ versus $45.4 \pm 12.15)$. This might be due to the difference in life expectancy in two countries and a large number of the study population. However, our previous institutional study shows similar age distribution among the patients with RA [33].

On the basis of our data, older RA patients with a long disease duration were more susceptible to comorbidity 


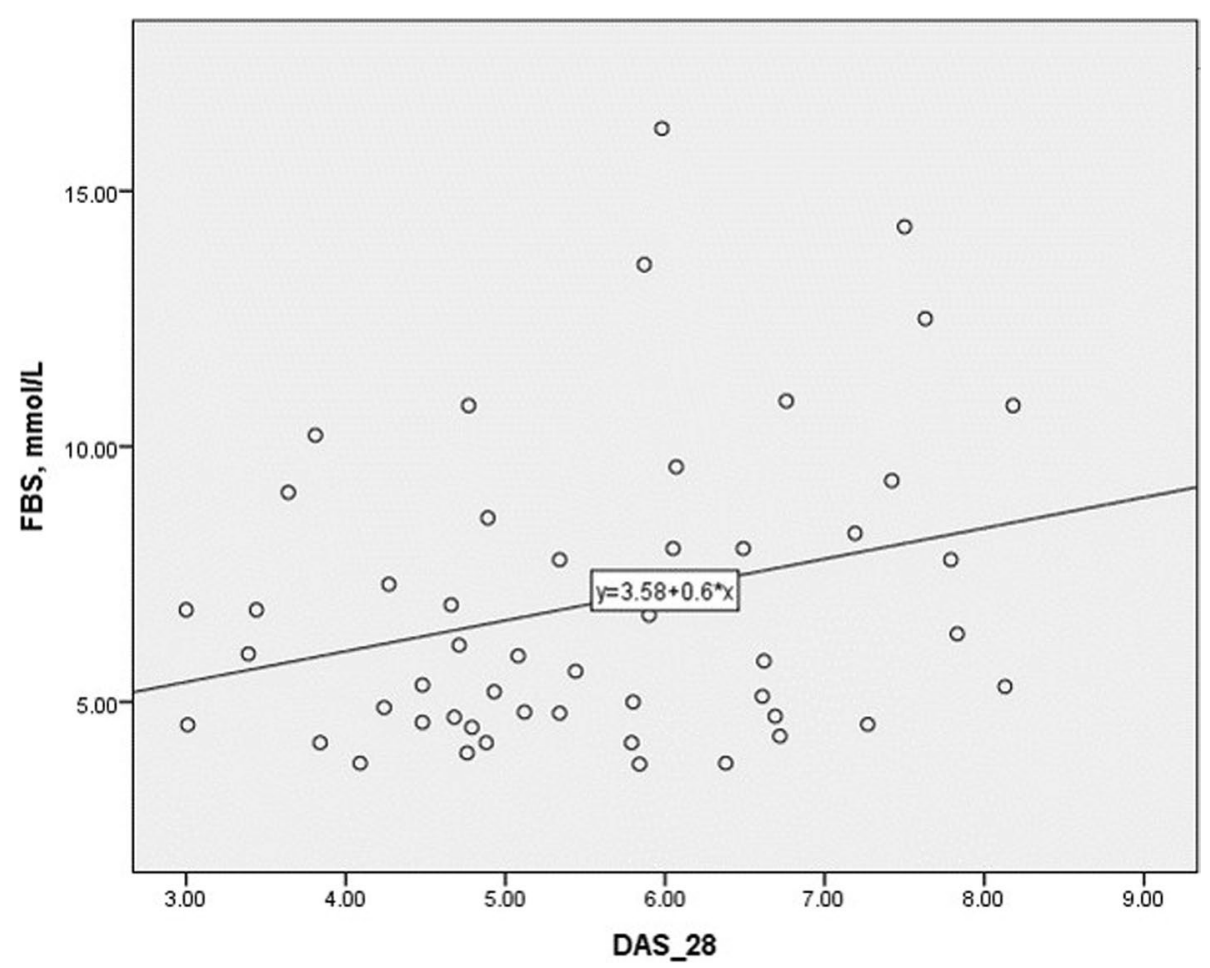

Fig. 2 Correlation between FBS and DAS-28 of the RA patients. FBS, fasting blood sugar; DAS-28, Disease Activity Score Including 28 Joints

with diabetes but the distinction was not statistically significant. To understand co-morbidity associations, the total joint damage and physiological dysfunction in patients with long-term RA durations is more likely to escalate because of a long-term inflammatory procedure, resulting in greater physical inactivity which could damage glucose metabolism [29]. Examination of about fifteen hundred RA cases with varying disease duration for certain comorbidities which includes diabetes revealed a $9.3 \%$ prevalence of diabetes reported by Ranganath VK et al. [34]. They also observed that aging is correlated substantially with comorbidity and prolonged disease span ( $p$ value 0.001) [34]. Initial assessment for RA and earlier care is highly critical in order to avoid more joint damage. After initiation of therapy, the first few months have important repercussions on the long-term prognosis [30]. Clinical experiments have shown that individuals with lower disease progression have a higher long-term result within 6 months of initiation of therapy.

Based on our study findings, we cannot draw a conclusion about the involvement of steroid therapy in developing DM since out of 150 patients, only 72 were receiving steroid during enrollment of this research, and no significant discrepancy was found in the prevalence of DM among steroid users and non-users $(p \leq 0.216)$. In patients with RA, steroids are widely used. While steroids traditionally increase the glucose level of plasma, the potentially anti-inflammatory action may increase the insulin secretion from pancreas cells and enhance the sensitivity of insulin in peripheral tissue [35]. No correlation was noticed by Toms TE et al. between steroid consumption for a prolonged time and higher frequency of metabolic syndrome in a cross-sectional sample of 398 RA patients. Dessein PH et al., however, have shown that the reduced insulin sensitivity in patients with RA has been associated with elevated oral prednisolone (cumulative $4.8 \mathrm{~g}$ (range 2.0-8.5)) and high doses of pulsed glucocorticoids [36, 37]. Early RA patients with short-term usage of steroids permit stronger regulation of the disease activities without a substantial increase in DM risk, which can be clarified by improved insulin tolerance, as proposed by Svenson KL et al. [22].

Diabetes prevalence in patients with RA as revealed by our study relative to the expected prevalence of DM in the Bangladeshi population was higher (36\%) than that (6.9\%) mentioned in the earlier report [https://www.idf.org/ our-network/regions-members/south-east-asia/membe rs/93-bangladesh.html]. Additionally, we observed 10\% 


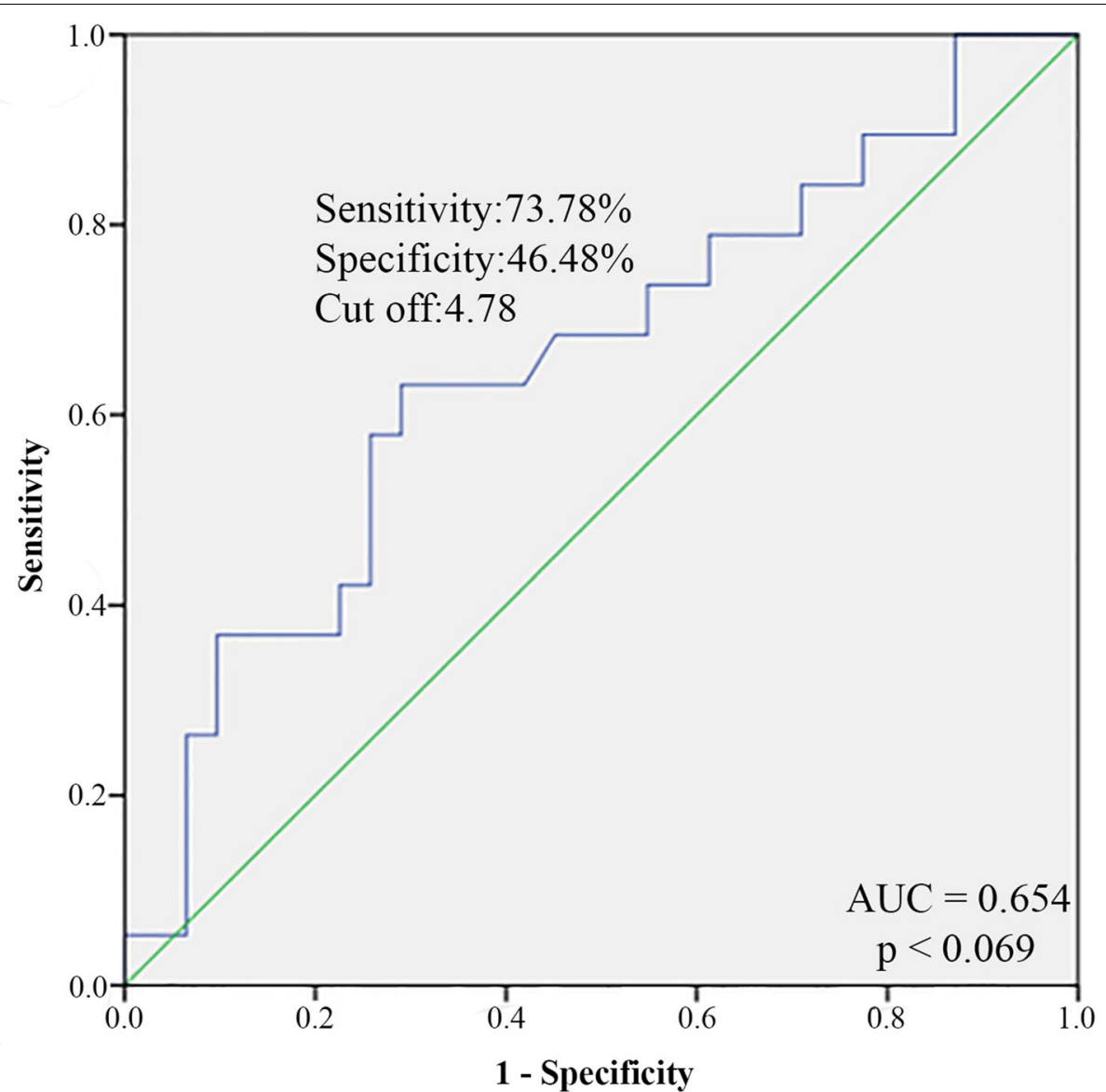

Fig. 3 Receiver operator characteristic (ROC) curve of mean value DAS-28 in predicting the development of type 2 diabetes (T2DM)

Table 6 Comparison between diabetic and nondiabetic patients

\begin{tabular}{llll}
\hline Variables & Diabetic $(\boldsymbol{n}=\mathbf{5 4})$ & $\begin{array}{l}\text { Non diabetic } \\
(\boldsymbol{n}=\mathbf{9 6})\end{array}$ & $\boldsymbol{P}$ value \\
\hline $\begin{array}{l}\text { Age in years (mean } \\
\mathbf{\pm} \text { SD) }\end{array}$ & $51.21 \pm 11.17$ & $41.84 \pm 11.47$ & $0.007^{*}$ \\
$\begin{array}{l}\text { Disease duration in } \\
\text { years }\end{array}$ & $5(1-10)$ & $1.5(0.25-5)$ & 0.056 \\
Steroid use, $\boldsymbol{n}$ (\%) & $30(55.6 \%)$ & $35(36.5 \%)$ & 0.216 \\
$\begin{array}{l}\text { Tender joint count } \\
\text { Swollen joint count }\end{array}$ & $6(0-8)$ & $10(6-16)$ & 0.075 \\
ESR & $35(32-65)$ & $32(18-48)$ & 0.087 \\
CRP & $20(12.49)$ & $21.9(3.6-46)$ & 0.234 \\
DAS-28 & $6.05(4.77-7.42)$ & $5.68(4.48-6.38)$ & 0.569 \\
\hline
\end{tabular}

Data are expressed as median (interquartile range) if not otherwise mentioned *Statistically significant

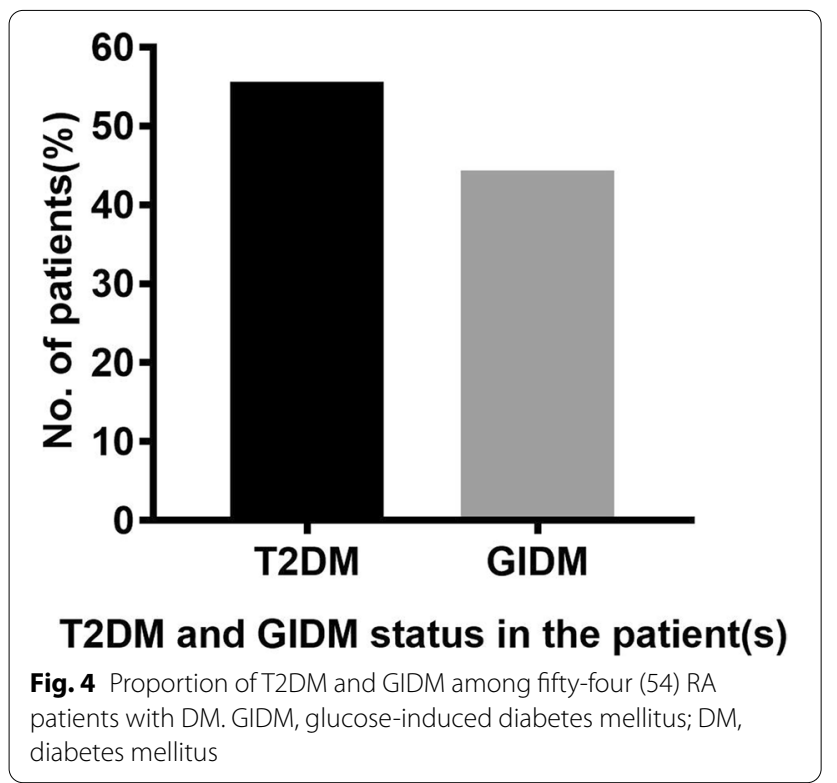


of patients displaying T2DM in RA patients, confirming the available literature [38]. A transitional abnormality in T2DM natural history is IFG which may provide an important and cost-effective mechanism for cardio-metabolic risk stratification in theory [39]. To date, however, no research has verified IFG's predictive function in RA patients.

\section{Conclusions}

We conclude that elderly RA patients are more susceptible to DM, however, a large multicenter, control group cohort analysis is needed to conclude a genuine association between RA and DM. Given the higher pervasiveness of DM in RA patients, we suggest evaluating for DM regularly, implying that either HbA1C or OGTT are estimated on account of determination and afterward yearly in all RA patients. Clinicians ought to be aware of the burden of diabetes and prediabetes not being distinguished in RA patients. Additionally, essential cardiovascular and diabetes preventive counseling for RA patients should be accentuated for the curative procedure. Moreover, our findings offer the basis for clinical intervention approaches for RA care and support the potential advancement of precise medication.

\begin{abstract}
Abbreviations
ACPA: Anticyclic citrullinated peptide antibodies; ADA: American Diabetes Association; BMI: Body mass index; CSC: Corticosteroids; CMCH: Chittagong Medical College Hospital; CRP: C-reactive protein; CV: Cardiovascular; CVE: Cardiovascular eEvents; DAS-28: Disease Activity Score Including 28 Joints; ESR: Erythrocyte sedimentation rate; EULAR: European League Against Rheumatism; FPG: Fasting plasma glucose; GIDM: Glucose-induced diabetes mellitus; IFG: Impaired fasting glucose; IGT: Impaired glucose tolerance; IL: Interleukin; IQR: Intraquartile range; MeTs: Metabolic syndrome; OGTT: Oral glucose tolerance test; PPG: Postprandial glucose; RA: Rheumatoid Arthritis; RF: Rheumatoid factor; SJC: Swollen joints ount; T2D: Type 2 diabetes; TJC: Tender joints count; TNF: Tumor necrosis factor; 2HA75gm Glucose: 2 hours after $75 \mathrm{gm}$ glucose.
\end{abstract}

\section{Acknowledgements}

We would like to thank all the members of the Medicine Department, Chittagong Medical College and Hospital, Chattogram-4203, Bangladesh, for their cordial support.

\section{Authors' contributions}

PKP, Al, MSAH, ASMM, JD, BC, SD, DC, MFFC, MSA, AY, and PKN collected the data. PKP, Al, and MJI performed the statistical analysis. The figures and tables were prepared by PKP, Al, and MSAH. PKP and Al conceived the study and designed the experimental procedures. Al supervised the study. PKP, Al, and MSAH wrote the manuscript. All authors read and approved the manuscript. PKP and Al have contributed equally in the study.

\section{Funding}

No funding sources.

\section{Availability of data and materials}

The dataset used and/or analyzed during the current study is available from the corresponding author on reasonable request.

\section{Declarations}

\section{Ethics approval and consent to participate}

The Ethical Review Board of Chittagong Medical College, Chittagong-4203, Bangladesh, approved our study protocol (Ref./CMC/PG/2017/373). Participation was voluntary and consent of each participant was taken privately, prior to sample collection by the issuance of a consent form. All answers were kept confidential and refusal to take part or withdrawal from the study did not hamper his/her treatment.

\section{Consent for publication}

Not applicable.

\section{Competing interests}

The authors declare that they have no competing interests.

\section{Author details}

${ }^{1}$ Chittagong Medical College, 57 K.B. Fazlul Kader Rd, Chattogram 4203, Bangladesh. ${ }^{2}$ Mawlana Bhashani Science and Technology University, Santosh, Tangail 1902, Bangladesh. ${ }^{3}$ University of Chittagong, Chittagong University Rd, Chattogram 4331, Bangladesh

Received: 4 September 2021 Accepted: 4 November 2021

Published online: 17 December 2021

\section{References}

1. Scott DL, Wolfe F, Huizinga TW (2010) Rheumatoid arthritis. Lancet 376:1094-1108

2. Giacomelli R, Gorla R, Trotta F, Tirri R, Grassi W, Bazzichi L, Galeazzi M, Matucci-Cerinic M, Scarpa R, Cantini F (2015) Quality of life and unmet needs in patients with inflammatory arthropathies: results from the multicentre, observational RAPSODIA study. Rheumatology 54(5):792-797

3. Maetzel A, Li L, Pencharz J, Tomlinson G, Bombardier C (2004) The economic burden associated with osteoarthritis, rheumatoid arthritis, and hypertension: a comparative study. Ann Rheum Dis 63(4):395-401

4. Kwoh C, Anderson L, Greene J, Johnson D, O'Dell J, Robbins M (2002) Guidelines for the management of rheumatoid arthritis: 2002 update. Arthritis Rheum 46:328-346

5. Schoels M, Knevel R, Aletaha D, Bijlsma JW, Breedveld FC, Boumpas DT, Burmester G, Combe B, Cutolo M, Dougados M (2010) Evidence for treating rheumatoid arthritis to target: results of a systematic literature search. Ann Rheum Dis 69(4):638-643

6. Dougados M, Soubrier M, Antunez A, Balint P, Balsa A, Buch MH, Casado G, Detert J, El-Zorkany B, Emery P (2014) Prevalence of comorbidities in rheumatoid arthritis and evaluation of their monitoring: results of an international, cross-sectional study (COMORA). Ann Rheum Dis 73(1):62-68

7. Gabriel SE (2008) Why do people with rheumatoid arthritis still die prematurely? Ann Rheum Dis 67(Suppl 3):iii30-iii34

8. Gabriel SE, Michaud K (2009) Epidemiological studies in incidence, prevalence, mortality, and comorbidity of the rheumatic diseases. Arthritis Res Ther 11(3):1-16

9. Wolfe F, Mitchell DM, Sibley JT, Frieds JF, Bloch DA, Williams CA, Spitz PW, Haga M, Kleinheksel SM, Cathey MA (1994) The mortality of rheumatoid arthritis. Arthritis Rheum 37:481-494

10. Association AD (2009) Diagnosis and classification of diabetes mellitus. Diabetes Care 32(Supplement 1):S62-S67

11. Jiang P, Li H, Li X (2015) Diabetes mellitus risk factors in rheumatoid arthritis: a systematic review and meta-analysis. Clin Exp Rheumatol 33(1):115-121

12. Han C, Robinson DW, Hackett MV, Paramore LC, Fraeman KH, Bala MV (2006) Cardiovascular disease and risk factors in patients with rheumatoid arthritis, psoriatic arthritis, and ankylosing spondylitis. J Rheumatol 33(11):2167-2172

13. Mutru O, Laakso M, Isomäki H, Koota K (1989) Cardiovascular mortality in patients with rheumatoid arthritis. Cardiology 76(1):71-77 
14. Myllykangas-Luosujärvi R, Aho K, Kautiainen H, Isomäki H (1995) Cardiovascular mortality in women with rheumatoid arthritis. J Rheumatol 22(6):1065-1067

15. Prior P, Symmons D, Scott D, Brown R, Hawkins C (1984) Cause of death in rheumatoid arthritis. Rheumatology 23(2):92-99

16. Allebeck $P$ (1982) Increased mortality in rheumatoid arthritis: the use of a medical information system for assessment of death risks. Scand J Rheumatol 11(2):81-86

17. Festa A, D'Agostino R Jr, Howard G, Mykkanen L, Tracy RP, Haffner SM (2000) Chronic subclinical inflammation as part of the insulin resistance syndrome: the Insulin Resistance Atherosclerosis Study (IRAS). Circulation 102(1):42-47

18. Xu H, Barnes GT, Yang Q, Tan G, Yang D, Chou CJ, Sole J, Nichols A, Ross JS, Tartaglia LA (2003) Chronic inflammation in fat plays a crucial role in the development of obesity-related insulin resistance. J Clin Invest 112(12):1821-1830

19. Pradhan AD, Manson JE, Rifai N, Buring JE, Ridker PM (2001) C-reactive protein, interleukin 6, and risk of developing type 2 diabetes mellitus. Jama 286(3):327-334

20. Schmidt MI, Duncan BB, Sharrett AR, Lindberg G, Savage PJ, Offenbacher S, Azambuja MI, Tracy RP, Heiss G, Investigators A (1999) Markers of inflammation and prediction of diabetes mellitus in adults (Atherosclerosis Risk in Communities study): a cohort study. Lancet 353(9165):1649-1652

21. Barzilay Jl, Abraham L, Heckbert SR, Cushman M, Kuller LH, Resnick HE, Tracy RP (2001) The relation of markers of inflammation to the development of glucose disorders in the elderly: the Cardiovascular Health Study. Diabetes 50(10):2384-2389

22. Svenson $\mathrm{KL}$, Pollare T, Lithell $H$, Hällgren R (1988) Impaired glucose handling in active rheumatoid arthritis: relationship to peripheral insulin resistance. Metabolism 37(2):125-130

23. Dessein PH, Stanwix AE, Joffe BI (2002) Cardiovascular risk in rheumatoid arthritis versus osteoarthritis: acute phase response related decreased insulin sensitivity and high-density lipoprotein cholesterol as well as clustering of metabolic syndrome features in rheumatoid arthritis. Arthritis Res 4(5):R5. https://doi.org/10.1186/ar428

24. Dessein PH, Joffe BI, Stanwix A, Botha AS, Moomal Z (2002) The acute phase response does not fully predict the presence of insulin resistance and dyslipidemia in inflammatory arthritis. J Rheumatol 29(3):462-466

25. Charles P, Elliott MJ, Davis D, Potter A, Kalden JR, Antoni C, Breedveld FC, Smolen JS, Eberl G, deWoody K (1999) Regulation of cytokines, cytokine inhibitors, and acute-phase proteins following anti-TNF-a therapy in rheumatoid arthritis. J Immunol 163(3):1521-1528

26. Beckham JC, Caldwell DS, Peterson BL, Pippen AM, Currie MS, Keefe FJ, Weinberg JB (1992) Disease severity in rheumatoid arthritis: relationships of plasma tumor necrosis factor-a, soluble interleukin 2-receptor, soluble CD4/CD8 ratio, neopterin, and fibrin D-dimer to traditional severity and functional measures. J Clin Immunol 12(5):353-361

27. Antohe JL, Bili A, Sartorius JA, Kirchner HL, Morris SJ, Dancea S, Wasko MCM (2012) Diabetes mellitus risk in rheumatoid arthritis: reduced incidence with anti-tumor necrosis factor a therapy. Arthritis Care Res 64(2):215-221

28. Dessein $\mathrm{PH}$, Tobias M, Veller MG (2006) Metabolic syndrome and subclinical atherosclerosis in rheumatoid arthritis. J Rheumatol 33(12):2425-2432

29. Emamifar A, Levin K, Jensen Hansen IM (2017) Patients with newly diagnosed rheumatoid arthritis are at increased risk of diabetes mellitus: an observational cohort study. Acta Reumatol Port 42(4):310-317

30. Solomon DH, Karlson EW, Rimm EB, Cannuscio CC, Mandl LA, Manson JE, Stampfer MJ, Curhan GC (2003) Cardiovascular morbidity and mortality in women diagnosed with rheumatoid arthritis. Circulation 107(9):1303-1307

31. Akter S, Rahman MM, Abe SK, Sultana P (2014) Prevalence of diabetes and prediabetes and their risk factors among Bangladeshi adults: a nationwide survey. Bull World Health Organ 92:204-213A

32. Akhtar S, Nasir JA, Sarwar A et al (2020) Prevalence of diabetes and prediabetes in Bangladesh: a systematic review and meta-analysis. BMJOpen 10:e036086

33. Rahman M, Lohani KH, Nath RK, Hasan MU, Paul S, Rahman MH, Faruk G, Uddin MN, Biswas RSR, Karim MR (2018) Efficacy of methotrexate in combination with antioxidant vitamins (A, C \& E) versus methotrexate alone in the treatment of rheumatoid arthritis. Open Forensic Sci J 3(1)
34. Ranganath VK, Maranian P, Elashoff DA, Woodworth T, Khanna D, Hahn T, Sarkisian C, Kremer JM, Furst DE, Paulus HE (2013) Comorbidities are associated with poorer outcomes in community patients with rheumatoid arthritis. Rheumatology 52(10):1809-1817

35. Wasko MC, Kay J, Hsia EC, Rahman MU (2011) Diabetes mellitus and insulin resistance in patients with rheumatoid arthritis: risk reduction in a chronic inflammatory disease. Arthritis Care Res 63(4):512-521

36. Dessein PH, Joffe BI, Stanwix AE, Christian BF, Veller M (2004) Glucocorticoids and insulin sensitivity in rheumatoid arthritis. J Rheumatol 31(5):867-874

37. Toms TE, Panoulas VF, Douglas KM, Griffiths HR, Kitas GD (2008) Lack of association between glucocorticoid use and presence of the metabolic syndrome in patients with rheumatoid arthritis: a cross-sectional study. Arthritis Res Ther 10(6):1-8

38. Ursini F, Russo E, D'Angelo S, Arturi F, Hribal ML, D'Antona L, Bruno C, Tripepi G, Naty S, De Sarro G (2016) Prevalence of undiagnosed diabetes in rheumatoid arthritis: an OGTT study. Medicine 95(7)

39. Zhang X, Imperatore G, Thomas W, Cheng YJ, Lobelo F, Norris K, Devlin HM, Ali MK, Gruss S, Bardenheier B (2017) Effect of lifestyle interventions on glucose regulation among adults without impaired glucose tolerance or diabetes: a systematic review and meta-analysis. Diabetes Res Clin Pract 123:149-164

\section{Publisher's Note}

Springer Nature remains neutral with regard to jurisdictional claims in published maps and institutional affiliations. 